\title{
Multiple Endemic Solutions in an Epidemic Hepatitis B Model without Vertical Transmission*
}

\author{
Nelson Owuor Onyango \\ School of Mathematics, CBPS, University of Nairobi, Nairobi, Kenya \\ Email: nelsonowuor@gmail.com, onyango@uonbi.ac.ke
}

Received 12 July 2014; revised 13 August 2014; accepted 23 August 2014

Copyright (C) 2014 by author and Scientific Research Publishing Inc.

This work is licensed under the Creative Commons Attribution International License (CC BY). http://creativecommons.org/licenses/by/4.0/

(c) (i) Open Access

\section{Abstract}

This paper examines the dynamics of Hepatitis B via a Susceptible Exposed Infectious Recovered (SEIR) type epidemic model. Previous studies have shown that Hepatitis B is characterized by multiple endemic solutions, a matter which may be of concern in developing control strategies. We identify the possible causes of multiple endemic solutions in a Hepatitis B model and conclude that the dependance of the probability of carriage development $(q(\Lambda))$ on the force of infection $(\Lambda)$ is the main reason for multiple endemicity. Other factors such as a large proportion of infants that are not vaccinated $(\omega)$ may also enhance the possibility of multiple endemicity. The role of carriers may also play a key role in the possibility of such complex dynamics, i.e., when infectiousness of carriers- $(\alpha)$ is high, the probability of existence of multiple endemic equilibrium solutions is increased. In our arguments, the traditional reproduction number $R_{0}<1$ which we define here by a function $G(0)<1$ does not imply stability of disease-free equilibrium.

\section{Keywords}

SEIR Epidemic Models, Endemic Equilibriums, Hepatitis B

\section{Introduction}

Multiple endemicity (often arising from a backward bifurcation) refers to a situation where a dynamic system

"Modeling multiple endemicity in Hepatistis B: This research work was motivated by a result of a previous work published by Medley G. in 2001, showing simulated results of multiple endemicity for Hepatitis B. 
(ODE or PDE) converges in its asymptotic dynamics to two different equilibrium states, under the same set of parameter values. The theory of attractors and mutation of such attractors with change in parameter values is studied extensively under the field of Bifurcation Theory [1] [2].

The dynamics of Hepatitis B (HepB) virus can be classified into three major world prevalence regions [3]:

- Sub-Sahara Africa where horizontal infection among lower age groups is the dominant form of transmission, together with perinatal transmission (mother to child transmission within the first year of the infant). The region is hyper-endemic (over 10\% carrier prevalence) since more infection among children leads to higher carrier prevalence.

- South East Asia where vertical transmission is reportedly more significant. Then other forms of childhood infection also contribute. This region is hyper-endemic as well.

- Europe and North America where only transmission among adults contributes to infection. Sexual contact and intra-venous drug use are the key contact pathways. The region is low-endemic as very few adults develop to carrier state after acute infection.

We model a scenario that captures the Sub-Saharan prevalence zone: where vertical transmission could play a role in transmission; immunization largely done at child birth without screening and mainly heterosexual behavior is assumed in the population. Although HepB can be effectively vaccinated against, there are publichealth concerns we wish to address that may affect the optimal vaccination against the disease and global control strategies. Recent developments in the dynamics of HepB virus indicate existence of complex dynamics such as multiple endemicity, heterogenous prevalence among population groups, among others, which may be of great concern to vaccination efforts.

This paper aims to investigate the following scenarios in the context of a developing country (Sub-Sahara Africa scenario):

- Causes for existence of multiple endemic scenario in an age-structured model. This phenomenon has been observed in a model without age structure [4]. Multiple endemicity may offer a great challenge to vaccination campaign, since even in cases when vaccination is effective, the possibility of coexistence of higher and lower disease endemic states may complicate a vaccination effort aimed at disease control.

- The threshold conditions for HepB endemicity. The reproduction number $G_{0}<1$ does not imply the stability of disease-free equilibrium in this case.

The paper is organized into the following sections: Section 1 offers an introduction and justification for modeling HepB. In Section 2, we describe the model structure and the equations for the population dynamics. In Section 3, we consider the model of proportions which is also referred to as the projected model due to the fact that it captures equilibrium dynamics in terms of the vital (birth and death) parameters. In Section 4, we derive a fixed-point equation using the equation for the force of infection and characterize the equilibrium solutions via this fixed-point equation. Section 5, the multiple endemicity conditions are obtained for this case of a HepB model without vertical transmission.

\section{Model Structure}

We develop an age-structured compartmental model with the following compartments: $S(a, t)$ are susceptible individuals; $L(a, t)$ are latently infected individuals who are infected but not yet infectious; $I(a, t)$ are acute infected individuals who are quite infectious. But they move on quickly to the next state, the chronic carriage state, so they do not contribute strongly to new infections; $C(a, t)$ are carriers who in some literature (see e.g. [5]) are referred to as chronic infectious individuals. They stay in this state for a long time and contribute more to new infections than the acute infectious group; and $R(a, t)$ are the immune or else referred to as recovered individuals.

A better view of the model structure is displayed using the Figure 1 and Figure 2.

The following parameters define movement between the compartments: $\Lambda(a, t)$ is the force of infection which varies with age and time; $\sigma$ defines the rate of movement from latent state to infectious state; $q(\Lambda(a, t))$ is the probability that an individual who is infectious becomes a carrier, so $(1-q(\Lambda(a, t)))$ is the probability that such a person becomes immune; $\gamma_{1}, \gamma_{2}$ are recovery rates from infectious and carrier states, respectively; $b(a)$ is the age-dependent birth rate, only used in the boundary conditions into $S(a, t)$, $L(a, t)$ and $R(a, t)$ states; $\mu(a)$ denotes the age-dependent mortality rate.

Define the total population for an age group by 


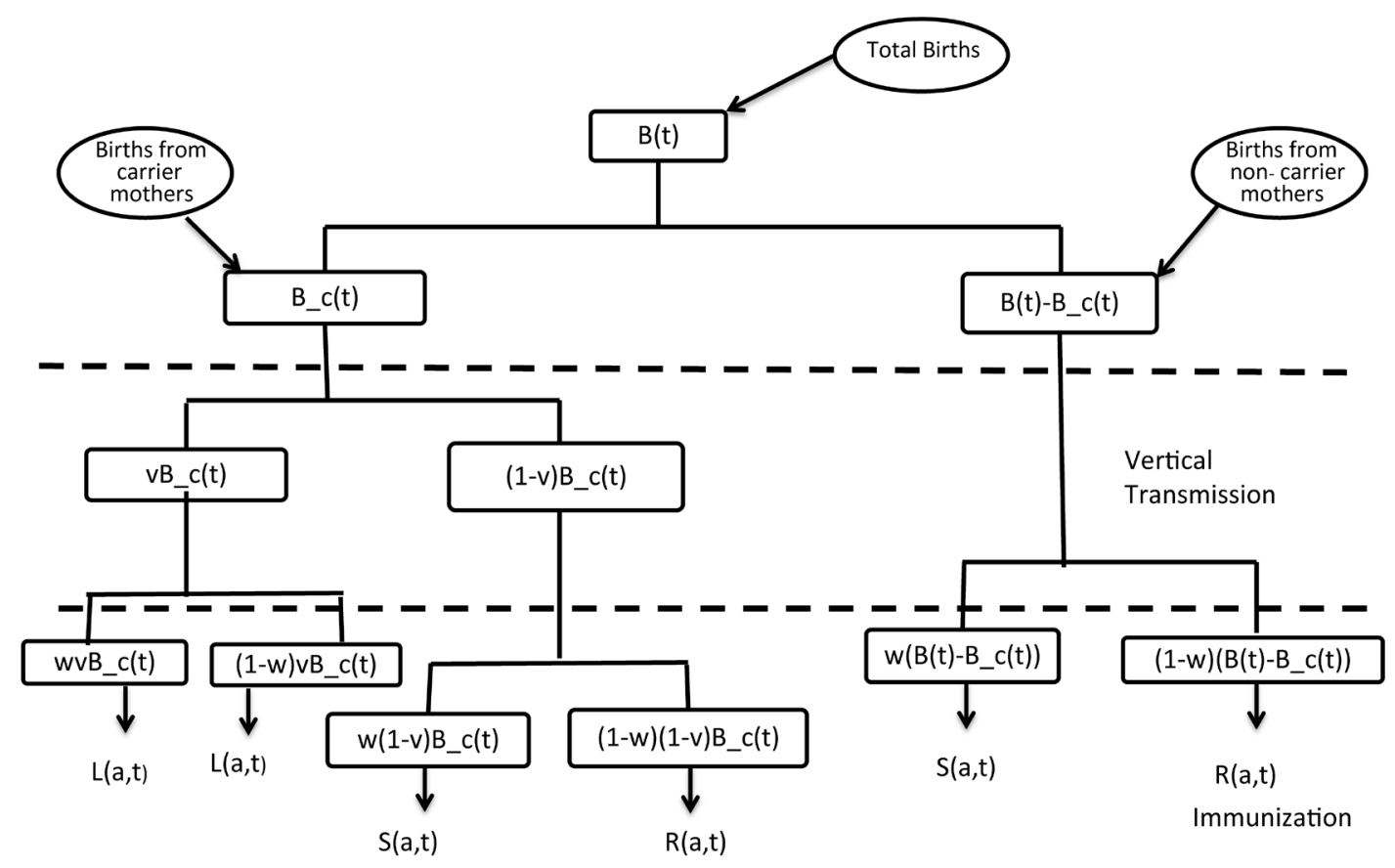

Figure 1. The figure show proportions of new births into population compartments, where $B(t)$ denotes new births in numbers, $v, w$ represent vertical transmission and immunization parameters respectively, $(1-w)$ represents total vaccination coverage while $L(a, t), S(a, t)$ and $R(a, t)$ are population compartments as defined within the text.

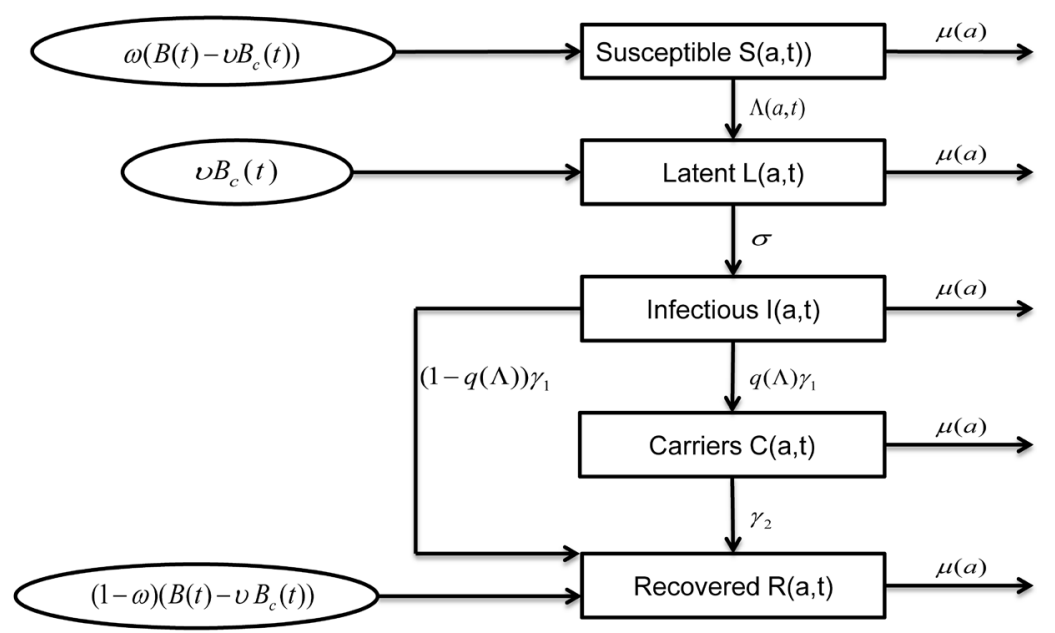

Figure 2. Flow chart of HepB virus transmission.

$$
N(a, t)=S(a, t)+L(a, t)+I(a, t)+C(a, t)+R(a, t)
$$

and $B(t)$ as the total number of new births at time $t$ by

$$
B(t)=\int_{0}^{\bar{a}} N(a, t) b(a) \mathrm{d} a,
$$

while $B_{c}(t)$, the total number of births from carrier mothers by

$$
B_{c}(t)=\int_{0}^{\bar{a}} C(a, t) b(a) \mathrm{d} a .
$$


Therefore $\left\{B(t)-B_{c}(t)\right\}$ represents births from non-carrier mothers. We generally assume that significant vertical transmission occurs mainly from carrier mothers and a very small proportion (that we can ignore) of vertical transmission come from acute infected mothers. The model also assumes that vaccination is done to newborn only.

Given $\omega$ as the proportion of births that are not successfully immunized, $(1-\omega)$ defines immunization coverage for newborn children. We suppose also that a proportion $v$ of newborn babies from carrier mothers get infected (vertical transmission) and thus a proportion $(1-v)$ of children do not acquire infection from the mother (at birth). Children who acquire infection through vertical transmission enter the population at the latent stage and soon develop into the acute infected individuals. This is logical since it is rare that children are newly born with symptoms and signs of any disease they may have acquired from the mother during pregnancy or at birth.

We therefore have the following increments into $S(a, t), L(a, t)$ and $R(a, t)$ classes via new births, which define the boundary conditions in the model.

New births into the susceptible class are given by

$$
\omega\left\{(1-v) B_{c}(t)+\left(B(t)-B_{c}(t)\right)\right\}=\omega\left\{B(t)-v B_{c}(t)\right\}=\omega\left\{\int_{0}^{\bar{a}} b(a) N(a, t) \mathrm{d} a-v \int_{0}^{\bar{a}} b(a) C(a, t) \mathrm{d} a\right\},
$$

into the latent class by

$$
\omega v B_{c}(t)+(1-\omega) v B_{c}(t)=v B_{c}(t)=v \int_{0}^{\bar{a}} b(a) C(a, t) \mathrm{d} a
$$

and into the immune class by

$$
\begin{aligned}
(1-\omega)(1-v) B_{c}(t)+(1-\omega)\left\{B(t)-B_{c}(t)\right\} & =(1-\omega)\left\{B(t)-v B_{c}(t)\right\} \\
& =(1-\omega)\left\{\int_{0}^{\bar{a}} b(a) N(a, t) \mathrm{d} a-v \int_{0}^{\bar{a}} b(a) C(a, t) \mathrm{d} a\right\} .
\end{aligned}
$$

We use the notation

$$
\left(\partial_{t}+\partial_{a}\right) S(a, t)=\left\{\frac{\partial}{\partial t}+\frac{\partial}{\partial a}\right\} S(a, t) .
$$

Under the assumptions above, we describe the full model for the spread of HepB by the system of PDEs below,

$$
\begin{aligned}
\left(\partial_{t}+\partial_{a}\right) S(a, t) & =-\Lambda(a, t) S(a, t)-\mu(a) S(a, t) \\
S(0, t) & =\omega\left\{\int_{0}^{\bar{a}} b(a) N(a, t) \mathrm{d} a-v \int_{0}^{\bar{a}} b(a) C(a, t) \mathrm{d} a\right\} \\
\left(\partial_{t}+\partial_{a}\right) L(a, t) & =\Lambda(a, t) S(a, t)-\sigma L(a, t)-\mu(a) L(a, t) \\
L(0, t) & =v \int_{0}^{\bar{a}} b(a) C(a, t) \mathrm{d} a \\
\left(\partial_{t}+\partial_{a}\right) I(a, t) & =\sigma L(a, t)-\gamma_{1} I(a, t)-\mu(a) I(a, t) \\
I(0, t) & =0 \\
\left(\partial_{t}+\partial_{a}\right) C(a, t) & =q(\Lambda(a, t)) \gamma_{1} I(a, t)-\gamma_{2} C(a, t)-\mu(a) C(a, t) \\
C(0, t) & =0 \\
\left(\partial_{t}+\partial_{a}\right) R(a, t) & =\gamma_{2} C(a, t)+\{1-q(\Lambda(a, t))\} \gamma_{1} I(a, t)-\mu(a) R(a, t) \\
R(0, t) & =(1-\omega)\left\{\int_{0}^{\bar{a}} b(a) N(a, t) \mathrm{d} a-v \int_{0}^{\bar{a}} b(a) C(a, t) \mathrm{d} a\right\},
\end{aligned}
$$

which we denote in matrix form by

$$
\begin{gathered}
\left(\partial_{t}+\partial_{a}\right) \boldsymbol{X}(a, t)=\boldsymbol{F}(\boldsymbol{X}(a, t))+\boldsymbol{A} \boldsymbol{X}(a, t)-\mu(a) \boldsymbol{I}_{5} \boldsymbol{X}(a, t) \\
\boldsymbol{X}(0, t)=[S(0, t), \cdots, R(0, t)]^{\mathrm{T}}
\end{gathered}
$$




$$
\boldsymbol{X}(a, 0)=\boldsymbol{X}_{0}(a)
$$

where $\boldsymbol{I}_{5}$ denotes a 5 by 5 identity matrix,

$$
\left.\begin{array}{rl}
\boldsymbol{X}(a, t)=(S(a, t), L(a, t), I(a, t), C(a, t), R(a, t))^{\mathrm{T}}, \\
-\Lambda(a, t) S(a, t) \\
\Lambda(a, t) S(a, t) \\
0 \\
q(\Lambda(a, t)) \gamma_{1} I(a, t) \\
(1-q(\Lambda(a, t))) \gamma_{1} I(a, t)
\end{array}\right),
$$

and

$$
\boldsymbol{A}=\left(\begin{array}{ccccc}
0 & 0 & 0 & 0 & 0 \\
0 & -\sigma & 0 & 0 & 0 \\
0 & 0 & -\gamma_{1} & 0 & 0 \\
0 & 0 & 0 & -\gamma_{2} & 0 \\
0 & 0 & 0 & \gamma_{2} & 0
\end{array}\right) .
$$

The total population has an exponential decay and is obtained by summing the variables in (1.4)

$$
\begin{gathered}
\left(\partial_{t}+\partial_{a}\right) N(a, t)=-\mu(a) N(a, t) \\
N(0, t)=\int_{0}^{\bar{a}} b(a) N(a, t) \mathrm{d} a \\
N(a, 0)=N_{0}(a) .
\end{gathered}
$$

System (2) is known as the stable population model and has a unique persistent age distribution profile (see e.g., [6]-[8])

$$
p(a):=\lim _{t \rightarrow \infty} \frac{N(a, t)}{\int_{0}^{\bar{a}} N(a, t) \mathrm{d} a} .
$$

\section{Projected Model}

The projected model represents the situation when the population dynamics are at their equilibrium. This state can be achieved by transforming the state variables into proportions, i.e., modify system (1.4) into a model of proportions. This transformation eliminates the vital dynamics (birth and death) from the model and hence the reason why we refer to the resultant model as the projected model.

Consider the proportions of the population in each compartment in the model at age $a$, time $t$ :

$$
\begin{aligned}
& s(a, t):=\frac{S(a, t)}{N(a, t)}, \quad l(a, t):=\frac{L(a, t)}{N(a, t)}, \quad i(a, t):=\frac{I(a, t)}{N(a, t)}, \\
& c(a, t):=\frac{C(a, t)}{N(a, t)}, \quad r(a, t):=\frac{R(a, t)}{N(a, t)}
\end{aligned}
$$

and

$$
\lim _{a \rightarrow \infty} p(a, t)=\lim _{a \rightarrow \infty} \frac{N(a, t)}{P(t)} \rightarrow p(a)
$$

where $P(t)=\int_{0}^{\bar{a}} N(a, t) \mathrm{d} a$ denotes the total population across all age groups. The projected susceptible population is thus obtained next. Applying the quotient rule on the derivative $\left(\partial_{t}+\partial_{a}\right) S(a, t) / N(a, t)$, we have, 


$$
\begin{aligned}
&\left(\partial_{t}+\partial_{a}\right) S(a, t) / N(a, t)=\frac{\left(\partial_{t}+\partial_{a}\right) S(a, t)}{N(a, t)}-\frac{S(a, t)}{N(a, t)} \cdot \frac{\left(\partial_{t}+\partial_{a}\right) N(a, t)}{N(a, t)} \\
&=\frac{-\Lambda(a, t) S(a, t)-\mu(a) S(a, t)}{N(a, t)}-\frac{S(a, t)}{N(a, t)} \cdot \frac{-\mu(a) N(a, t)}{N(a, t)} \\
&\left(\partial_{t}+\partial_{a}\right) s(a, t)=-\Lambda(a, t) s(a, t) .
\end{aligned}
$$

Define

$$
\hat{b}(a):=\frac{N(a, t) b(a)}{\int_{0}^{\bar{a}} N(a, t) b(a) \mathrm{d} a}, \quad \Omega:=v \int_{0}^{\bar{a}} c(a, t) \hat{b}(a) \mathrm{d} a
$$

Then the boundary condition for the susceptible class reads:

$$
\begin{aligned}
s(0, t) & =\frac{S(0, t)}{N(0, t)}=\frac{\omega \int_{0}^{\bar{a}} b(a) N(a, t) \mathrm{d} a}{\int_{0}^{\bar{a}} b(a) N(a, t) \mathrm{d} a}-\frac{\omega v \int_{0}^{\bar{a}} b(a) C(a, t) \mathrm{d} a}{\int_{0}^{\bar{a}} b(a) N(a, t) \mathrm{d} a} \\
& =\omega-\omega v \int_{0}^{\bar{a}} c(a, t) \frac{N(a, t) b(a)}{\int_{0}^{\bar{a}} b(a) N(a, t) \mathrm{d} a} \mathrm{~d} a \\
& =\omega\left\{1-v \int_{0}^{\bar{a}} c(a, t) \hat{b}(a) \mathrm{d} a\right\}=\omega(1-\Omega) .
\end{aligned}
$$

Proceeding the same way for all the state variables, we obtain the projected system (1.8), which is independent of population dynamics, except for birth rate that appears in the model within the boundary conditions. The projected system now reads:

$$
\begin{aligned}
\left(\partial_{t}+\partial_{a}\right) s(a, t) & =-\Lambda(a, t) s(a, t) ; \\
s(0, t) & =\omega(1-\Omega) \\
\left(\partial_{t}+\partial_{a}\right) l(a, t) & =\Lambda(a, t) s(a, t)-\sigma l(a, t) ; \\
l(0, t) & =\Omega \\
\left(\partial_{t}+\partial_{a}\right) i(a, t) & =\sigma l(a, t)-\gamma_{1} i(a, t) ; \\
i(0, t) & =0 \\
\left(\partial_{t}+\partial_{a}\right) c(a, t) & =q(\Lambda(a, t)) \gamma_{1} i(a, t)-\gamma_{2} c(a, t) ; \\
c(0, t) & =0 \\
\left(\partial_{t}+\partial_{a}\right) r(a, t) & =\gamma_{2} c(a, t)+(1-q(\Lambda(a, t))) \gamma_{1} i(a, t) ; \\
r(0, t) & =(1-\omega)(1-\Omega),
\end{aligned}
$$

which can be expressed in matrix form as

$$
\begin{gathered}
\left(\partial_{t}+\partial_{a}\right) \boldsymbol{x}(a, t)=\boldsymbol{F}(\boldsymbol{x}(a, t))+\boldsymbol{A} \boldsymbol{x}(a, t) \\
\boldsymbol{x}(0, t)=[\omega(1-\Omega), \Omega, 0,0,(1-\omega)(1-\Omega)]^{\mathrm{T}} \\
\boldsymbol{x}(a, 0)=\boldsymbol{x}_{0}(a)
\end{gathered}
$$

where $x(a, t)=s(a, t)+s(a, t)+l(a, t)+i(a, t)+c(a, t)+r(a, t)$ and

$$
\boldsymbol{F}(\boldsymbol{x}(a, t)):=\left(\begin{array}{c}
-\Lambda(a, t) s(a, t) \\
\Lambda(a, t) s(a, t) \\
0 \\
q(\Lambda(a, t)) \gamma_{1} i(a, t) \\
(1-q(\Lambda(a, t))) \gamma_{1} i(a, t)
\end{array}\right),
$$




$$
\boldsymbol{A}=\left(\begin{array}{ccccc}
0 & 0 & 0 & 0 & 0 \\
0 & -\sigma & 0 & 0 & 0 \\
0 & \sigma & -\gamma_{1} & 0 & 0 \\
0 & 0 & 0 & -\gamma_{2} & 0 \\
0 & 0 & 0 & \gamma_{2} & 0
\end{array}\right) .
$$

\section{Force of Infection}

The force of infection $\Lambda(a, t)$, which defines the rate at which susceptible individuals become infected, takes the form of standard incidence rate

$$
\Lambda(a, t)=\int_{0}^{\bar{a}} \frac{J(a, b)}{P(t)}\{I(b, t)+\alpha C(b, t)\} \mathrm{d} b .
$$

In this case, we have an additional infection from carriers, where the parameter $\alpha$ defines the infectiousness of carriers relative to acute infected group and $P(t)=\int_{0}^{\bar{a}} N(a, t) \mathrm{d} a$. The transmission coefficient

$J(a, b)=k(a) \beta(b)$, is where the contacts between seropositive individuals and susceptible individuals are weighted by the contacts in each group per unit time, this is the separable mixing case (see e.g. [9]-[11]). Other forms of transmission coefficient include constant case $J(a, b)=\beta \quad[10]$ and $\quad J(a, b)=\beta(b)$ [12].

We similarly obtain the force of infection for the projected model at equilibrium:

$$
\begin{aligned}
\Lambda(a) & =\int_{0}^{\bar{a}} k(a) \beta(b) \frac{1}{P(t)}\{I(b)+\alpha C(b)\} \mathrm{d} b=\int_{0}^{\bar{a}} k(a) \beta(b) \frac{N(b, t)}{P(t)}\{i(b)+\alpha c(b)\} \mathrm{d} b \\
& =\int_{0}^{\bar{a}} k(a) \beta(b) p(b)\{i(b)+\alpha c(b)\} \mathrm{d} b .
\end{aligned}
$$

In the ensuing discussions, we analyse a reduced model, i.e., the model without vertical transmission. Vertical transmission characterizes a situation where a fraction of newborns are born with the disease, having been infected before or at birth. In this setting, the parameter $v$ defines this fraction. If $v=0$, then we do not have vertical transmission. Recall that $v$ appears in the system (1.5) only via $\Omega:=v \int_{0}^{\bar{a}} c(a, t) \hat{b}(a) \mathrm{d} a$, defined in Equation (1.7).

The model without vertical transmission is thus,

$$
\begin{gathered}
\left(\partial_{t}+\partial_{a}\right) \boldsymbol{x}(a, t)=\boldsymbol{F}(\boldsymbol{x}(a, t))+\boldsymbol{A} \boldsymbol{x}(a, t) ; \\
\boldsymbol{x}(0, t)=[\omega, 0,0,0,(1-\omega)]^{\mathrm{T}} \\
\boldsymbol{x}(a, 0)=\boldsymbol{x}_{0}(a)
\end{gathered}
$$

For this subcase $(v=0)$, we know from a general case [13] that if the initial age distributions are in $L^{1}$ and are non-negative, then the solutions to system (1.12) exist globally, remain non-negative and stay in $L^{1}$. The semi-group methods are used to prove the well-possedness of the system. The case of existence of solutions when vertical transmission is present has also been worked out [6], again using semi-group methods.

We obtain expressions for $i(b)$ and $c(b)$ and insert in Equation (1.11).

Consider system (1.12) at its equilibrium such that,

$$
\frac{\mathrm{d} x(a)}{\mathrm{d} a}=\boldsymbol{F}(x(a))+\boldsymbol{A} x(a) .
$$

Define $h(a)=\exp \{-a\}$. Thus

$$
i(a)=\int_{0}^{a} \omega \sigma \frac{h\left(\gamma_{1} a\right)}{h\left(\gamma_{1} \tau_{1}\right)} \int_{0}^{\tau_{1}} \frac{h\left(\sigma \tau_{1}\right)}{h\left(\sigma \tau_{0}\right)} \Lambda\left(\tau_{1}\right) \exp \left(-\int_{0}^{\tau_{0}} \Lambda\left(a^{*}\right) \mathrm{d} a^{*}\right) \mathrm{d} \tau_{0} \mathrm{~d} \tau_{1}
$$

and 


$$
c(a)=\omega \sigma \gamma_{1} \int_{0}^{a} \frac{h\left(\gamma_{2} a\right)}{h\left(\gamma_{2} \tau_{2}\right)} q(\Lambda(a)) \int_{0}^{\tau_{2}} \frac{h\left(\gamma_{2} \tau_{2}\right)}{h\left(\gamma_{1} \tau_{1}\right)} Q(*) \mathrm{d} \tau_{1} \mathrm{~d} \tau_{2}
$$

where

$$
Q(*)=\int_{0}^{\tau_{1}} \frac{h\left(\sigma \tau_{1}\right)}{h\left(\sigma \tau_{0}\right)} \Lambda\left(\tau_{1}\right) \exp \left(-\int_{0}^{\tau_{0}} \Lambda\left(a^{*}\right) \mathrm{d} a^{*}\right) \mathrm{d} \tau_{0} .
$$

Inserting these expressions in Equation (1.11), we obtain

$$
\Lambda(a)=: \tilde{G}(\Lambda(a))
$$

By the definition of the force of infection, one observes that $\tilde{G}(\Lambda)$ is a next generation operator, an operator that defines new infective individuals coming from an initial density of infective individuals. The operator $\tilde{G}(\Lambda)$ is best be understood via its eigenvalues. We offer a definition of the Basic Reproduction Number as we shall use it in this context.

Definition 1. The Basic Reproduction Number is defined as the spectral radius of the operator $\tilde{G}(\Lambda)$ [14]; that is,

$$
R_{0}=\rho(\tilde{G}(\Lambda(a)))=\lim _{m \rightarrow \infty}\left\|\tilde{G}(\Lambda(a))^{m}\right\|^{1 / m},
$$

the m-th generation of new infective individuals coming from an initial density of infective individuals.

The Basic Reproduction number $R_{0}$ has been shown to have its shortcomings [15]. We also discuss an anomaly on the conventional use of $R_{0}$ for the HepB model that we have studied.

Theorem 1. Assume that the force of infection $\Lambda$ has age dependance only via the state variables, such that $\Lambda(a)=\Lambda$. Then Equation (1.19) disintegrates into

$$
\Lambda=\Lambda G(\Lambda) .
$$

where

$$
G(\Lambda)=\left\{\omega \hat{K}_{1}\left[\exp \left\{-\Lambda \tau_{0}\right\}\right]+\alpha q(\Lambda) \omega \hat{K}_{2}\left[\exp \left\{-\Lambda \tau_{0}\right\}\right]\right\} .
$$

Proof. We make the following transformations on Equation (1.13): $\tilde{l}(a)=l(a) / \Lambda, \tilde{i}(a)=i(a) / \Lambda$, and $\tilde{c}(a)=c(a) / \Lambda$, thus removing $\Lambda$ dependance in all equations except $s(a)$.

We seek solutions $i(a)$ and $c(a)$ and later insert back in Equation (1.11). For notational convenience, we define $K_{j}: L_{+}^{1} \rightarrow \mathbb{R}_{+}: j=(1,2)$ and $\hat{K}_{j}: L_{+}^{1} \rightarrow \mathbb{R}_{+}: j=(1,2) \quad \Phi:=\exp \left(-\Lambda \tau_{0}\right)$ such that

$$
\begin{aligned}
& K_{1}[\Phi]:=\int_{0}^{a} \frac{h\left(\gamma_{1} a\right)}{h\left(\gamma_{1} \tau_{1}\right)} \sigma \int_{0}^{\tau_{1}} \frac{h\left(\sigma \tau_{1}\right)}{h\left(\sigma \tau_{0}\right)} \Phi \mathrm{d} \tau_{0} \mathrm{~d} \tau_{1} \\
& K_{2}[\Phi]:=\int_{0}^{a} \sigma \gamma_{1} \frac{h\left(\gamma_{2} a\right)}{h\left(\gamma_{2} \tau_{1}\right)} \int_{0}^{\tau_{2}} \frac{h\left(\gamma_{2} \tau_{2}\right)}{h\left(\gamma_{1} \tau_{1}\right)} \int_{0}^{\tau_{1}} \exp \left\{-\sigma\left(\tau_{1}-\tau_{0}\right)\right\} \frac{h\left(\sigma \tau_{1}\right)}{h\left(\sigma \tau_{0}\right)} \Phi \mathrm{d} \tau_{0} \mathrm{~d} \tau_{1} \mathrm{~d} \tau_{2} \\
& \hat{K}_{1}[\Phi]:=k(a) \int_{0}^{\bar{a}} \beta(b) p(b) K_{1}[\Phi] \mathrm{d} b \\
& \hat{K}_{2}[\Phi]:=k(a) \int_{0}^{\bar{a}} \alpha \beta(b) p(b) K_{2}[\Phi] \mathrm{d} b .
\end{aligned}
$$

The solutions $\tilde{i}(a)$ and $\tilde{c}(a)$ are

$$
\tilde{i}(a)=\omega \sigma \int_{0}^{a} \frac{h\left(\gamma_{1} a\right)}{h\left(\gamma_{1} \tau_{1}\right)} \int_{0}^{\tau_{1}} \frac{h\left(\sigma \tau_{1}\right)}{h\left(\sigma \tau_{0}\right)} \exp \left(-\Lambda \tau_{0}\right) \mathrm{d} \tau_{0} \mathrm{~d} \tau_{1}=\omega K_{1}[\Phi]
$$

and

$$
\tilde{c}(a)=\omega \sigma \gamma_{1} \int_{0}^{a} \frac{h\left(\gamma_{2} a\right)}{h\left(\gamma_{2} \tau_{2}\right)} q(\Lambda) \int_{0}^{\tau_{2}} \frac{h\left(\gamma_{2} \tau_{2}\right)}{h\left(\gamma_{1} \tau_{1}\right)} \int_{0}^{\tau_{1}} \frac{h\left(\sigma \tau_{1}\right)}{h\left(\sigma \tau_{0}\right)} \exp \left(-\Lambda \tau_{0}\right) \mathrm{d} \tau_{0} \mathrm{~d} \tau_{1} \mathrm{~d} \tau_{2}=\omega \sigma \gamma_{1} K_{2}[\Phi] .
$$

Hence 


$$
\begin{aligned}
\Lambda & =\Lambda k(a) \int_{0}^{\bar{a}} \beta(b) p(b)\left\{\omega K_{1}[\Phi]+\alpha q(\Lambda) \omega K_{2}[\Phi]\right\} \mathrm{d} b \\
& =\Lambda\left\{\omega \hat{K}_{1}[\Phi]+\alpha q(\Lambda) \omega \hat{K}_{2}[\Phi]\right\}=: \Lambda G(\Lambda) .
\end{aligned}
$$

The endemic solutions (non-trivial solutions) satisfy $G(\Lambda)=1$, where

$$
G(\Lambda)=\omega \hat{K}_{1}\left[\exp \left\{-\Lambda \tau_{0}\right\}\right]+\alpha q(\Lambda) \omega \hat{K}_{2}\left[\exp \left\{-\Lambda \tau_{0}\right\}\right]
$$

and we define the Basic Reproduction Number of the system (1.4) by [9]

$$
G(0)=\omega \hat{K}_{1}[1]+\alpha q(0) \omega \hat{K}_{2}[1] .
$$

Remark 1. The equation

$$
\Lambda=\Lambda G(\Lambda)
$$

is thus the main determinant for dynamics of the system (1.12). Solutions to system (1.12) correspond to solutions to the fixed-point equation. $\Lambda=0$ corresponds to the disease-free state of system (1.12) while $1=G(\Lambda)$ has at least one real positive solution $\Lambda^{*} \in \mathbb{R}_{+}$, which corresponds to the endemic equilibrium of system (1.12).

Remark 2. Proposition (1) has been applied in studies of equilibrium dynamics, stability and bifurcation in epidemic models (see e.g. [6] [9] [13]. In particular, it has been applied to study the question of backward bifurcation in [16]-[18].

\section{Multiple Endemicity}

We seek to establish conditions under which exactly two endemic solutions may occur.

Lemma 1. $\lim _{\Lambda \rightarrow \infty} G(\Lambda)=0$.

Proof.

$$
G(\Lambda)=\omega \hat{K}_{1}[\Phi](a)+\alpha q(\Lambda) \omega \hat{K}_{2}[\Phi](a) .
$$

Since $\Phi:=\exp \left\{-\Lambda \tau_{0}\right\}, \hat{K}_{i}: i=(1,2)$ are both decreasing as $\Lambda$ becomes large and thus $G(\Lambda)$ decays exponentially fast in $\Lambda$ towards zero, irrespective of the form that $q(\Lambda)$ takes. However, $q(\Lambda)$ defines the probability of development of carriage and is bounded in the interval [0,1]. Increase in $G(\Lambda)$ may only hold for some initial values of $\Lambda$, but the influence is inconsequential as $\Lambda$ becomes large.

Theorem 2. Assume $q(\Lambda)$ is increasing in $\Lambda$ such that $G(\Lambda)$ is uni-modal for $\Lambda \in \mathbb{R}_{+}$Then $G(\Lambda)=1$ has two solutions if

(i) $G(0)<1$.

(ii) there exists $\Lambda^{*}>0$, such that $G\left(\Lambda^{*}\right)>1$.

(iii) $\lim _{\Lambda \rightarrow \infty} G(\Lambda)=0$.

Corollary 1. If conditions in Theorem (2) hold, then the Equation (1.12) has exactly two endemic solutions if

(i) $\omega \hat{K}_{1}[1]+\omega q(0) \alpha \hat{K}_{2}[1]<1$.

(ii) there exists one $\Lambda^{*}>0$, such that $q\left(\Lambda^{*}\right)>\frac{(1-G(0))+\omega \hat{K}_{1}\left[1-\exp \left\{-\Lambda^{*} \tau_{0}\right\}\right]+\omega q(0) \alpha \hat{K}_{2}[1]}{\omega \alpha \hat{K}_{2}\left[1-\exp \left\{-\Lambda^{*} \tau_{0}\right\}\right]}$.

Proof. Since

$$
G(\Lambda)=\omega \hat{K}_{1}\left[\exp \left\{-\Lambda \tau_{0}\right\}\right]+\alpha q(\Lambda) \omega \hat{K}_{2}\left[\exp \left\{-\Lambda \tau_{0}\right\}\right]
$$

then $G(0)<1$ implies

$$
G(0)=\omega \hat{K}_{1}[1]+\omega q(0) \alpha \hat{K}_{2}[1]<1 .
$$

If there exists $\Lambda^{*}>0$ such that $G\left(\Lambda^{*}\right)>1$, then

$$
G\left(\Lambda^{*}\right)=\left\{\omega \hat{K}_{1}\left[\exp \left\{-\Lambda^{*} \tau_{0}\right\}\right]+\alpha q\left(\Lambda^{*}\right) \omega \hat{K}_{2}\left[\exp \left\{-\Lambda^{*} \tau_{0}\right\}\right]\right\}>1 .
$$

Making $q\left(\Lambda^{*}\right)$ the subject of interest, we obtain 


$$
q\left(\Lambda^{*}\right)>\frac{(1-G(0))+\omega \hat{K}_{1}\left[1-\exp \left\{-\Lambda^{*} \tau_{0}\right\}\right]+\omega q(0) \alpha \hat{K}_{2}[1]}{\omega \alpha \hat{K}_{2}\left[1-\exp \left\{-\Lambda^{*} \tau_{0}\right\}\right]} .
$$

Remark 3. We make the following two useful remarks:

1. $G(\Lambda)=1$ will attain two solutions if and only if $q(\Lambda)$ has a direct dependance on $\Lambda$. Otherwise, from Equation (1.20), we can observe that if $q(\Lambda)$ constant in $\Lambda$, then $G(\Lambda)$ is monotone decreasing in $\Lambda$ and we cannot have multiple endemicity.

2. One also observes the following relationships between $q(\Lambda)$ and

(i) $\omega$ : the non-vaccinated proportion of the population.

(ii) $\alpha$ : the infectiousness of carriers.

To make this observation, we re-write Equation (1.22) in the two forms below:

$$
\begin{aligned}
q\left(\Lambda^{*}\right) & >\frac{A_{1}}{\alpha A_{2}}+A_{3} . \\
& >\frac{B_{1}}{\omega B_{2}}+B_{3} .
\end{aligned}
$$

where $A_{i}$ and $B_{i}(i=1,2,3)$ are constants in $\alpha$ and $\omega$ respectively. The inequality in Equation (1.22) is reinforced for large values of $\alpha$ and $\omega$. This means that, if the carrier population is large and many new infections occur from the carrier population ( $\alpha$ is large), then the Equation (1.12) is likely to have multiple dynamics. Similarly, if a large proportion of the population is not vaccinated ( $\omega$ is large), then again, the condition for multiple endemicity is likely to hold.

3. The model with vertical transmission shall be considered in further work. The model is illustrated below. Consider Equation (1.8) at its equilibrium, i.e., no time dependance. We also assume that vertical transmission occurs at birth, i.e., $(v \neq 0)$. Finally we ignore the $\Lambda$ dependance on age, i.e., $\Lambda(a)=\Lambda$. Denote

$$
\begin{aligned}
& \Omega=v \int_{0}^{\bar{a}} c(a) \hat{b}(a) \mathrm{d} a . \\
& \frac{\mathrm{d}}{\mathrm{d} a} s(a)=-\Lambda s(a) ; \\
& s(0)=\omega(1-\Omega) \\
& \frac{\mathrm{d}}{\mathrm{d} a} l(a)=\Lambda s(a)-\sigma l(a) ; \\
& l(0)=\Omega \\
& \frac{\mathrm{d}}{\mathrm{d} a} \mathrm{i}(a)=\sigma l(a)-\gamma_{1} i(a) \text {; } \\
& i(0)=0 \\
& \frac{\mathrm{d}}{\mathrm{d} a} c(a)=q(\Lambda) \gamma_{1} i(a)-\gamma_{2} c(a) ; \quad c(0)=0 \\
& \frac{\mathrm{d}}{\mathrm{d} a} r(a)=\gamma_{2} c(a)+(1-q(\Lambda)) \gamma_{1} i(a) \text {; } \\
& r(0)=(1-\omega)(1-\Omega)
\end{aligned}
$$

\section{Conclusions}

Multiple endemicity, arising from a backward bifurcation is a phenomenon that has been studied in a number of epidemic models [19] [20]. Studies have also shown similar dynamics with HepB for some set of realistic parameter values [4]. Multiple endemicity has been observed mainly in models of HepB especially when the carrier prevalence is high. Besides, HepB has also been shown to be characterized by heterogenous dynamics such as heterogenous prevalence (1\% to $90 \%)$ [4]. Such complex dynamics may affect the ability to design a global control strategy to deal with HepB. Generally speaking, HepB is one of the most significant STIs especially in the South East Asia and Africa. Global figures show that viral Hepatitis is 10 times more common than HIV infection despite the fact that a global viral hepatitis treatment program is still a long way from becoming a reality [21]. Vaccination and antiviral treatments are available but rare especially in developing countries. 
We studied the solutions of an SEIR HepB model and their stability via the force of infection. This approach has been used to determine equilibrium solutions and their stability in age-structured models, (see e.g., [9]). We make use of this approach to characterize the conditions for multiple endemic states from a fixed-point equation derived from an equation for the force of infection.

We investigated the role of the following parameters in determining the dynamics of the disease, in line with results of [4]:

- $q(\Lambda)=$ probability of development of carriage;

- $c(a, t)=$ prevalence of carriers;

- $\alpha=$ infectiousness of carriers;

- $(1-\omega)=$ immunization rate.

In further work, we shall incorporate the parameter $v$ for vertical transmission and explore its possible role in enhancing the probability of multiple endemicity. Our results are similar to those in [4], where the main cause of multiple endemicity is the dependance of the probability of development of carriage on the force of infection. This parameter dependance or inter-relationship has not been considered explicitly in many published models of HepB. But due to its role, it makes much sense to always consider this relationship. We also observe that the infectiousness of carriers may be key in enhancing possibilities of multiple endemicity. All in all, our results stress the key role that carriers play in the persistence of HepB within communities.

\section{Acknowledgements}

This work was done at the Institute of Biometry and Biomathematics, Helmholtz Centre-Munich, Germany, under the German DAAD support grant. The work was partly done during a postdoctoral visit at SLU in Uppsala, courtesy of a grant by the International Science Program (ISP)-Uppsala, Sweden. I wish to thank the University of Nairobi for granting me leave to finish the work.

\section{Funding}

Funding was partly from German DAAD postgraduate support grant and Swedish ISP Program (Uppsala University).

\section{References}

[1] Kuznetsov, A.Y. (2004) Elements of Applied Bifurcation Theory. 3rd Edition, Springer, Berlin. http://dx.doi.org/10.1007/978-1-4757-3978-7

[2] Guckenheimer, J. and Holmes, P. (1983) Nonlinear Oscillations, Dynamical Systems and Bifurcations of Vector Fields. Springer, Berlin. http://dx.doi.org/10.1007/978-1-4612-1140-2

[3] Edmunds, W.J., Medley, G.F. and Nokes, D.J. (1996) The Transmission Dynamics and Control of Hepatitis-B Virus in the Gambia. Statistics in Medicine, 15, 2215-2233. http://dx.doi.org/10.1002/(SICI)1097-0258(19961030)15:20<2215::AID-SIM369>3.0.CO;2-2

[4] Medley, G.F., Lindop, N.A., Edmunds, W.J. and Nokes, D.J. (1996) Hepatitis-B Virus Endemicity: Heterogeneity, Catastrophic Dynamics and Control. Nature Medicine, 7, 916-624.

[5] Zhao, S., Xu, Z. and Lu, Y. (2000) A Mathematical Model of Hepatitis B Virus Transmission and Its Application for Vaccination Strategy in China. International Journal of Epidemiology, 29, 744-752. http://dx.doi.org/10.1093/ije/29.4.744

[6] Inaba, H. (2006) Mathematical Analysis of an Age Structured SIR Epidemic Model with Vertical Transmission. Discrete and Continuous Dynamical Systems, Series B, 6, 69-96. http://dx.doi.org/10.3934/dcdsb.2006.6.69

[7] Webb, G.F. (1985) Theory of Non-Linear Age Dependent Population Dynamics. Marcel Dekker Inc., New York.

[8] Pruess, J. and Schappacher, W. (1984) Semigroup Methods for Age-Structured Population Dynamics. In: Chatterji, S., Fuchstainer, B., Kulisch, U. and Liedl, R., Eds., Jarbuch Überblicke Mathematik, Viewing Verlag.

[9] Thieme, H.R. (2003) Mathematics in Population Biology. Princeton University Press, New Jersey.

[10] Dietz, K. and Schlenze, D. (1985) Mathematical Models for Infectious Disease Statistics, a Celebration of Statistics. In: Atkinson, A.C. and Fienberg, S.E., Eds., The ISI Centenary Volume, Springer-Verlag, New York.

[11] Müller, J. (1998) Optimal Vaccination Patterns in Age-Structured Populations. SIAM Journal on Applied Mathematics, 59, 222-241. http://dx.doi.org/10.1137/S0036139995293270 
[12] Grippenberg, G. (1983) On a Non-Linear Intergral Equation Modeling an Epidemic in an Age-Structured Population. Journal for Pure and Applied Mathematics, 341, 56-67.

[13] Inaba, H. (1990) Threshold and Stability Results for an Age-Structured Epidemic Model. Journal of Mathematical Biology, 28, 411-434. http://dx.doi.org/10.1007/BF00178326

[14] Diekmann, O., Heesterbeek, J.A.P. and Metz, J.A.J. (1990) On the Definition and the Computation of the Basic Reproduction Ratio $R_{0}$ in Models for Infectious Diseases in Heterogeneous Populations. Journal of Mathematical Biology, 28, 365-382. http://dx.doi.org/10.1007/BF00178324

[15] Li, J., Blakeley, D. and Smith, R.J. (2011) The Failure of $R_{0}$. Computational and Mathematical Methods in Medicine, 2011, Article ID: 527610 .

[16] Chavez, C.C. and Song, B. (2004) Dynamical Models of Tuberculosis and Their Applications. Mathematical Bioscience and Engineering, 1, 361-404. http://dx.doi.org/10.3934/mbe.2004.1.361

[17] Hadeler, K.P. and Van den Driessche, P. (1997) Backward Bifurcation in Epidemic Control. Mathematical Biosciences, 146, 15-35. http://dx.doi.org/10.1016/S0025-5564(97)00027-8

[18] Sharomi, O., Podder, C.N., Gumel, A.B., Elbasha, E.H. and Watmough, J. (2000) Role of Incidence Function in Vaccine-Induced Backward Bifurcation in Some HIV Models. Mathematical Biosciences, 210, 436-463. http://dx.doi.org/10.1016/j.mbs.2007.05.012

[19] Garba, S.M., Gumel, A.B. and Bakar, M.R.A. (2008) Backward Bifurcations in Dengue Transmission Dynamics. Mathematical Biosciences, 215, 11-25. http://dx.doi.org/10.1016/j.mbs.2008.05.002

[20] Reluga, T.C., Medlock, J. and Perelson, A.S. (2008) Backward Bifurcations and Multiple Equilibria in Epidemic Models with Structured Immunity. Journal of Theoretical Biology, 252, 155-165. http://dx.doi.org/10.1016/j.jtbi.2008.01.014

[21] Robotin, M.C. (2011) Hepatitis B Prevention and Control: Lessons from the East and the West. World Journal of Hepatology, 3, 31-37. http://dx.doi.org/10.4254/wjh.v3.i2.31 
Scientific Research Publishing (SCIRP) is one of the largest Open Access journal publishers. It is currently publishing more than 200 open access, online, peer-reviewed journals covering a wide range of academic disciplines. SCIRP serves the worldwide academic communities and contributes to the progress and application of science with its publication.

Other selected journals from SCIRP are listed as below. Submit your manuscript to us via either submit@scirp.org or Online Submission Portal.
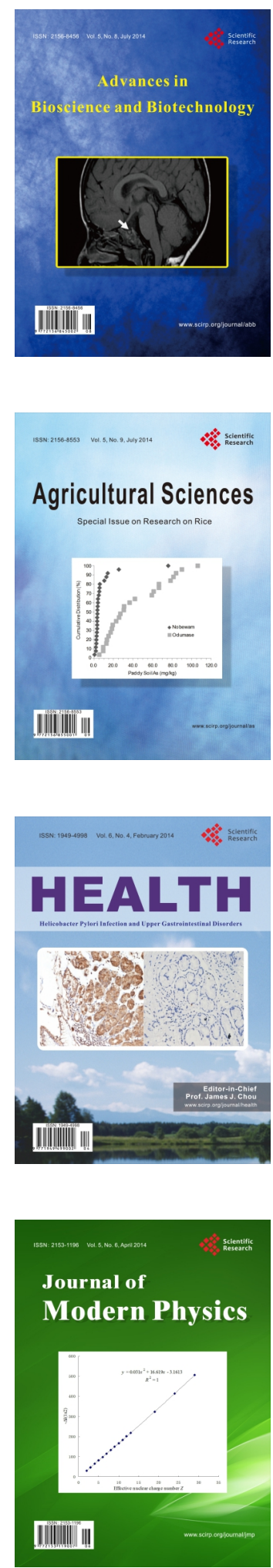
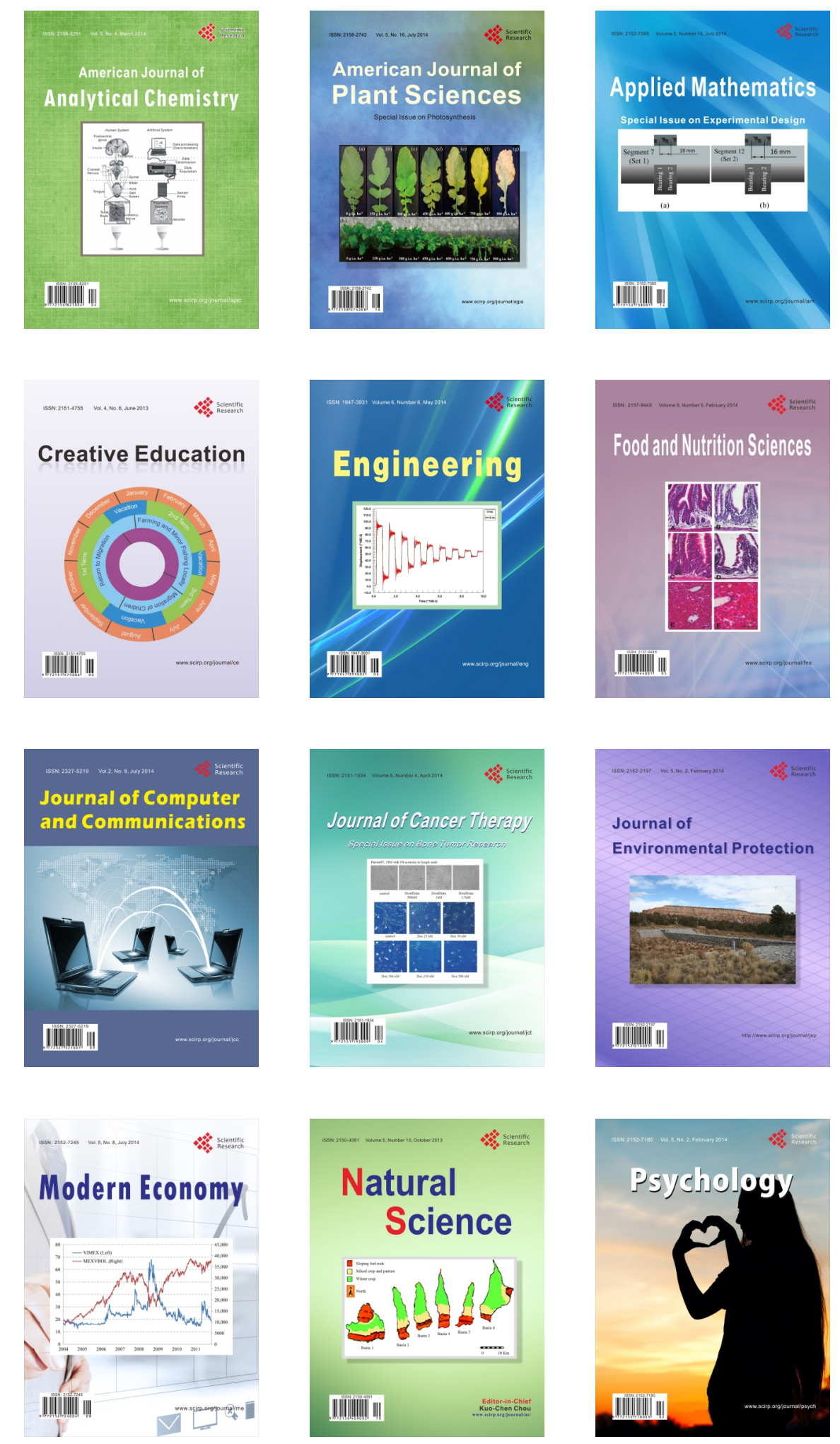